\title{
Folding and Spiralling: The Word View
}

\author{
Marcus Schaefer ${ }^{1}$ \\ CTI, DePaul University \\ Chicago, Illinois 60604
}

Eric Sedgwick ${ }^{2}$

CTI, DePaul University

Chicago, Illinois 60604

\section{Daniel Štefankovič ${ }^{3}$}

Computer Science Department

University of Rochester

Rochester, NY 14627-0226

\begin{abstract} Keywords: Spiralling, folding, string graphs.

\section{Introduction} producing psychedelic drawings like

$\overline{1 \text { Email: }}$ mschaefer@cti.depaul.edu

2 Email: esedgwick@cti.depaul.edu

3 Email: stefanko@cs.rochester.edu
\end{abstract}

We show that for every $n$ there are two simple curves on the torus intersecting at least $n$ times without the two curves folding or spiralling with respect to each other.

Maybe you have found yourself aimlessly doodling away on a piece of paper, 


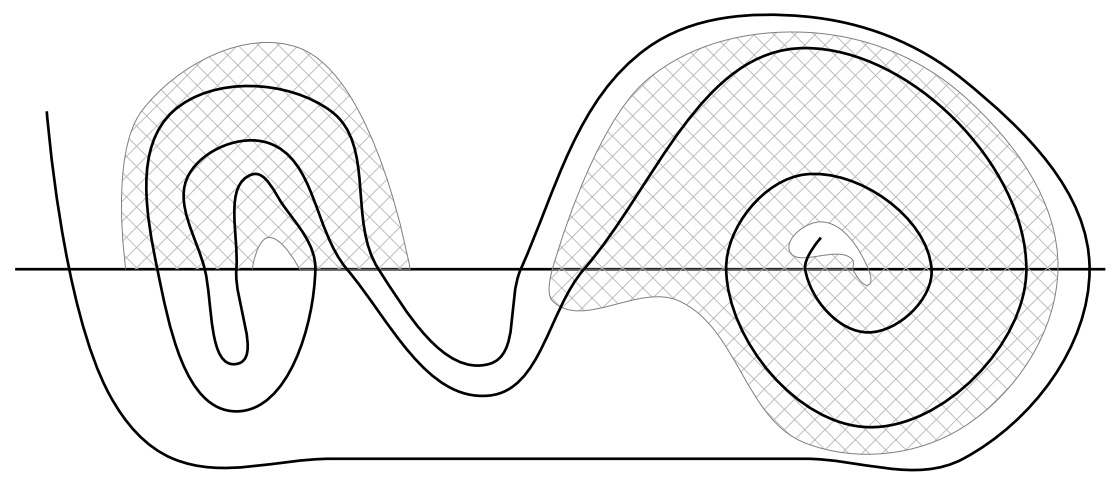

As you are squeezing one more bend of the curve into the picture you might have wondered whether the doodle has any inherent structure. The picture above, for example, contains a fair amount of spiralling and folding. If your curves intersect often, do they have to spiral or fold?

We need to make our notions of folding and spiralling precise. We are interested in the behavior of two simple curves, that is, curves without selfintersections. Typically, one of the curves is drawn as a straight line; it might be part of a triangulation of the surface we are working on. The second curve intersects the first curve a large number of times. We assume that the two curves do not form a lens, that is, a disc shaped area with two boundary components, one belonging to the first curve, the second to the other curve and not containing any other part of the curve in its interior. We can always eliminate a lens by punching a hole into the surface (the picture above contains four lenses which could be removed by adding two holes to the surface).

We say that two curves $\alpha$ and $\beta$ fold if there is a disk $D$ so that $\alpha$ coincides with the boundary $\partial D$ of the disk in two segments which have the same orientation (as we travel along $\alpha$ ), and $\beta$ intersects $\partial D$ only in those two segments $\partial D \cap \alpha$ and it does intersect both of those segments. The width of a fold is the number of intersections of $\beta$ with one of the two segments making up $\partial D \cap \alpha$ (both numbers have to be identical).

Two curves $\alpha$ and $\beta$ spiral if there is an annulus $A$ so that both curves have exactly one endpoint in common with each boundary component of $A$; moreover, we require that the spiral has depth at least one, where the depth of the spiral is the number of intersections of $\alpha$ and $\beta$ within $A$ minus 2 .

Our opening example contained a fold of width three and a spiral of depth one. (The disk $D$ and the annulus $A$ are crosshatched in the picture). The following example shows that in spite of an arbitrarily large number of intersections of $\alpha$ and $\beta$ there need not be any spiralling and only very shallow folding in the drawing: 


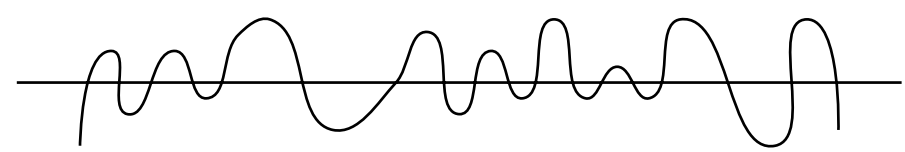

However, for this to be possible, there need to be a large number of boundary components. So let us assume that the surface is fixed, including the number of boundary components. Does a large number of intersections between $\alpha$ and $\beta$ on that surface force either a deep spiral or a wide fold?

This is not an entirely idle question inspired by research on the string graph problem. It is easy to show, adapting a construction due to Kratochvíl and Matoušek [3] for string graphs, that in the presence of $n$ boundary components two curves can be forced to intersect on the order of $\Omega\left(2^{n}\right)$ times in the plane without there being any spiralling. This example does, however, contain a fold of width $\Omega\left(2^{n}\right)$. In the companion paper to this paper we show that even for a fixed number of boundary components, we can construct curves $\alpha$ and $\beta$ in the plane with no spiralling and an arbitrarily large number of intersections between $\alpha$ and $\beta$ [8]. The two curves do form a wide fold, however.

In the present paper we show how to construct curves $\alpha$ and $\beta$ on the torus that contain no spiral and no fold. This closes a promising approach to the string graph problem on surfaces of higher genus. A string graph is the intersection graph of curves in the plane (or another surface). Recognizing whether a graph is a string graph is an old question $[1,2]$ that was settled recently by proving an exponential upper bound on the number of intersections needed in a realization of a string graph in the plane [4,9].

There are three known approaches to proving the decidability of string graphs in the plane: the topological proof by Pach and Tóth [4], our more combinatorial proof from [9] and an algebraic proof using trace monoids [6]. The last proof is the only one that currently works for arbitrary surfaces. Unfortunately, it only gives a double-exponential upper bound on the number of intersections in an optimal realization of a string graph on a surface. The proof from [9] makes essential use of properties of the plane, and does not seem to lift easily to other surfaces. This leaves the proof of Pach and Tóth which proceeds by finding, under certain conditions, a deep spiral in the realization of a string graph which then allows the simplification of the realization. Our main result of this paper implies that this approach cannot be lifted in a straightforward manner even to the torus, since there are systems of two curves that do not contain any spiralling whatsoever. 


\section{The Torus Case}

Let $f_{n}$ be the Fibonacci numbers, with $f_{0}=0$ and $f_{1}=1$. Figure 1 is a train track describing a system of curves on the torus.

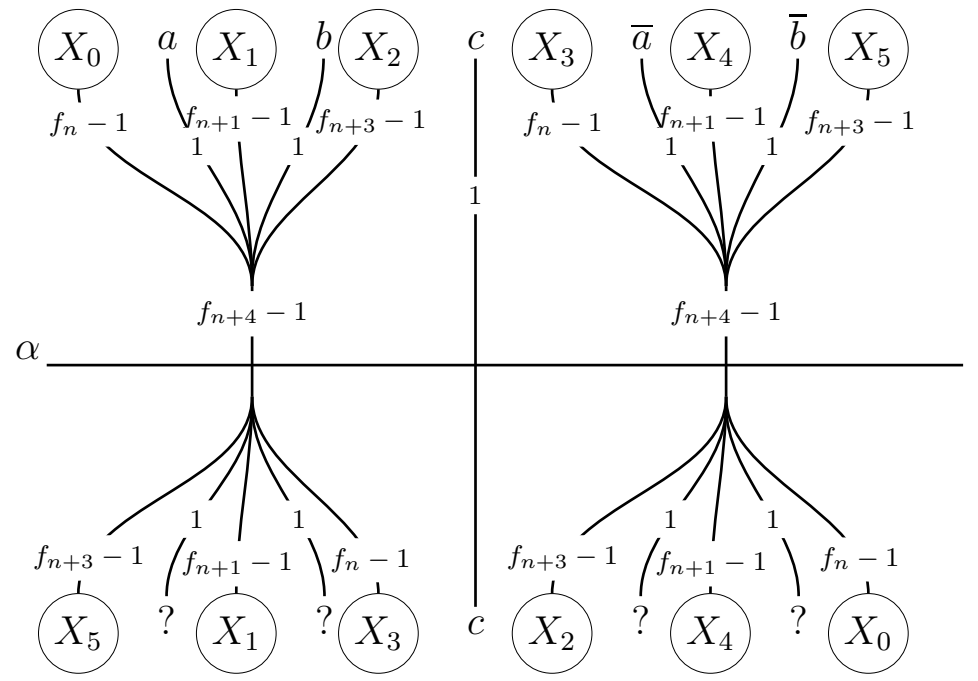

Fig. 1. Intersection of $\alpha$ with a system of curves containing no spirals.

A line of weight $w$ in the diagram represents $w$ parallel curves. For example, on the left side of Figure 1 we see five groups of parallel curves merge: the first group, labeled $X_{0}$, consists of $f_{n}-1$ parallel curves. We connect the groups that have the same label directly with each other, so, for example, the $f_{n}-1$ curves on the top left of the track connect to the occurrence of $X_{0}$ at the bottom right of the track. The connections can be realized simultaneously on a torus, so the whole system of curves can be embedded on the torus. Question marks represent unlabeled endpoints of curves.

It is not a priori clear that the train track describes a system of four curves labeled $a, b, \bar{a}$ and $\bar{b}$, and, indeed, it might not, depending on the value of $n$. However, we can prove that as long as $n \equiv 1 \bmod 3$, the train track represents a system of exactly four curves with endpoints and the system of curves does not contain any closed curve components.

We prove this result by viewing Figure 1 as a word equation which gives us the following system:

$$
X=X_{0} a X_{1} b X_{2} c X_{3} \bar{a} X_{4} \bar{b} X_{5}=X_{5} ? X_{1} ? X_{3} ? X_{2} ? X_{4} ? X_{0}
$$

with given lengths $\left|X_{0}\right|=\left|X_{3}\right|=F_{n}-1,\left|X_{1}\right|=\left|X_{4}\right|=F_{n+1}-1,\left|X_{2}\right|=$ $\left|X_{5}\right|=F_{n+3}-1$ (the question marks are unit-length variables).

We can show that for $n \equiv 1 \bmod 3$, Equation (1) has a unique solution. 
This solution translates into a system of four curves, $a, b, \bar{a}$ and $\bar{b}$. The fact that the solution is unique means that the system of curves does not contain any closed components.

A spiral in the train track forces a square in $X$, that is, a word of the form $w w$ with $w \in \Sigma^{*}$. By a rather intricate proof we can show that the unique solution $X$ to Equation (1) is square-free. Moreover, since a fold requires intersections with $\alpha$ in both directions (which does not occur in our system), we have established the following result.

Theorem 2.1 For any $n$ there are two simple curves $\alpha$ and $\beta$ on the torus which intersect more than $n$ times without forming either folding or spiralling.

\section{References}

[1] S. Benzer. On the topology of the genetic fine structure. Proceedings of the National Academy of Science, 45:1607-1620, 1959.

[2] Ron L. Graham. Problem 1. In Open Problems at 5th Hungarian Colloquium on Combinatorics, 1976.

[3] Jan Kratochvíl and Jiří Matoušek. String graphs requiring exponential representations. Journal of Combinatorial Theory, Series B, 53:1-4, 1991.

[4] János Pach and Géza Tóth. Recognizing string graphs is decidable. Discrete Comput. Geom., 28(4):593-606, 2002. Discrete and computational geometry and graph drawing (Columbia, SC, 2001).

[5] Marcus Schaefer, Eric Sedgwick, and Daniel Štefankovič. Algorithms for normal curves and surfaces. In Computing and combinatorics, volume 2387 of Lecture Notes in Comput. Sci., pages 370-380. Springer, Berlin, 2002.

[6] Marcus Schaefer, Eric Sedgwick, and Daniel Štefankovič. Recognizing string graphs in NP. J. Comput. System Sci., 67(2):365-380, 2003. Special issue on STOC2002 (Montreal, QC).

[7] Marcus Schaefer, Eric Sedgwick, and Daniel Štefankovič. Computing dehn twists and geometric intersection numbers in polynomial time. Technical Report TR05009, DePaul University, 2005.

[8] Marcus Schaefer, Eric Sedgwick, and Daniel Štefankovič. Spiralling and folding: The topological view. 2007.

[9] Marcus Schaefer and Daniel Štefankovič. Decidability of string graphs. $J$. Comput. System Sci., 68(2):319-334, 2004. 